\title{
Primary Amenorrhea Secondary to Mullerian Anomaly
}

Elif Sagsak, Asan Onder*, Fatma Doga Ocal, Yasemin Tasci, Sebahat Yilmaz Agladioglu, Semra Cetinkaya and Zehra Aycan

Dr. Sami Ulus Obstetrics and Gynecology, Pediatric Health and Disease Training and Research Hospital Pediatric Endocrinology Clinic, Turkey

\begin{abstract}
Mullerian developmental anomalies are rare causes of primary amenorrhea in $46, \mathrm{XX}$ adolescent girls. The aim to report this case is that Mullerian anomalies should be considered between the differential diagnosis of primary amenorrhea to prevent the delaying of the diagnosis. A 15 year-old female patient presented with a complaint of not menstruating. Medical history revealed an appendectomy at the age of 9 years, and surgical intervention due to a right para-ovarian hemorrhagic cyst at the age of 12 years. Apelvic Magnetic Resonance Imaging (MRI) evaluation revealed two uteri, one of which was rudimentary. Normal-sized uterus was not continued by vaginal lumen; however, the rudimentary uterus was connected with vaginal lumen. A hemorrhage to peritoneal cavity was suspected by pediatric endocrinologist and referred to gynecologist and radiologist for detailed investigation. It was concluded that the previously excised cyst might be bleeding into the peritoneal cavity as a result of menstruation. Then, the patient was scheduled for surgery.
\end{abstract}

Keywords: Mullerian anomaly; Amenorrhea; Adolescent

\section{Introduction}

While Mullerian duct anomalies cause many problems in women, such as infertility, recurrent miscarriage and disorders of sexual life, it can also present with amenorrhea during the adolescent period [1]. Mullerian anomalies include a wide range of anomalies from simple ones to complex ones. The diagnosis of Mullerian anomalies may be delayed till late puberty. These patients usually complain of primary amenorrhea and pelvic pain in adolescents. Although the exact incidence was not certain, it was approximately $0.1-2 \%$ in all women; $2-4 \%$ in infertile women, and $10-15 \%$ in cases of recurrent miscarriage [2]. Some studies reported the incidence rate in women as 7\% [3]. We present a case of primary amenorrhea associated with Mullerian anomaly.

\section{Case Report}

A 15-year-old adolescent girl presented with primary amenorrhea. Her past-medical history revealed an operation at the age of 9 years due to a perforated appendicitis, and a laparoscopic cyst excision with a diagnosis of right para-ovarian hemorrhagic cyst. Patient had complaints of chronic pelvic pain after the age of 11 years. The patient's family history was unremarkable. Her physical examination showed a final height of $146.5 \mathrm{~cm}(-2.5 \mathrm{SD})$, body weight of $51.5 \mathrm{~kg}(-0.4 \mathrm{SD})$, puberty Tanner stage 5, and there was not any other pathological finding. Her left-hand wrist radiograph showed that all epiphyses were closed. Laboratory tests revealed a Luteinizing Hormone (LH) level of $14.4 \mathrm{mIU} / \mathrm{mL}$, Follicle-Stimulating Hormone (FSH)level of $4.08 \mathrm{mIU} /$ $\mathrm{mL}$, estradiol (E2) level of $137 \mathrm{pg} / \mathrm{mL}$, and prolactin level of $7.99 \mathrm{ng} / \mathrm{dL}$ (normal range 1.9-25 ng/dL). Thyroid function test results were normal. Karyotype analysis revealed normal 46,XX. Pelvic ultrasonography (USG) revealed that the uterus and ovaries were at normal sizes with follicle cysts in the ovaries. A $35 \times 22 \mathrm{~mm}$ anechoic cyst was detected adjacent to the right ovary. Tumor markers Alpha-Fetoprotein (AFP), beta HCG (human chorionic gonadotropin), and Carcino Embryonic Antigen (CEA), which were evaluated following the detection of the paraovarian cyst, were within normal limits. However, serum cancer antigen 125 (CA-125) level (normal range $0-24 \mathrm{IU} / \mathrm{mL}$ ) was determined to behigh as it was63 IU/mL. Pelvic MRI revealed a rightward deviation of the uterus, pointed fundus, rudimentary cervix and significant vaginal hypoplasia. A rudimentary uterus with fluid in the $26 \times 10 \times 10$ $\mathrm{mm}$ cavity, which was not observed in the previous ultrasonographic evaluation, and cervix and vagina, connected to this uterus, extending to the introitus was found. A $5 \times 4 \times 3-\mathrm{cm}$ cystic lesion with high protein content was observed adjacent to the right ovary (Figures 1 and 2). The MRI findings of the patient were evaluated by an expert pediatric radiologist and an expert obstetrician. Their interpretation of MRI revealed two uteri, one of which was rudimentary. Normal-sized uterus was not continued by vaginal lumen; however, the rudimentary uterus was connected with vaginal lumen. It was believed that the normalsized uterus not continued by vaginal lumen wasopened into the peritoneal cavity It was believed that the patient was thus unable to menstruate due to her Mullerian anomaly and it was also concluded that the hemorrhagic cyst whichwas emptied at the age of 12 years was due to the menstrual bleeding into the peritoneal cavity. The patient was scheduled for surgery.

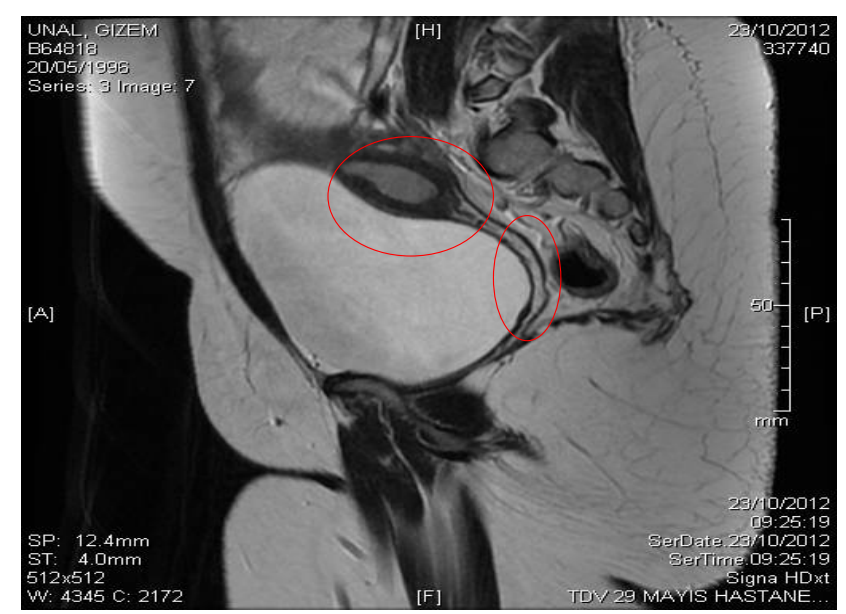

Figure 1: Normal and rudimentary uterus.

*Corresponding author: Asan Onder, Dr Sami Ulus Obstetrics and Gynecology, Pediatric Health and Disease Training and Research Hospital Pediatric Endocrinology Clinic, Turkey; Tel: +90 (312) 30565 08; Fax: +90(312) 31703 53; E-mail: asanonder@yahoo.com

Received March 09, 2014; Accepted March 27, 2014; Published March 31, 2014

Citation: Sagsak E, Onder A, Ocal FD, Tasci Y, Agladioglu SY, et al. (2014) Primary Amenorrhea Secondary to Mullerian Anomaly. J Clin Case Rep S1: 007. doi:10.4172/2165-7920.S1-007

Copyright: $\odot 2014$ Sagsak E, et al. This is an open-access article distributed under the terms of the Creative Commons Attribution License, which permits unrestricted use, distribution, and reproduction in any medium, provided the original author and source are credited. 


\section{Discussions}

Mullerian developmental anomalies are rare congenital anomalies. Multifactorial polygenic factors have been held responsible for their etiology. Familial descent has rarely been reported. Most cases have been de novo $[1,2]$.

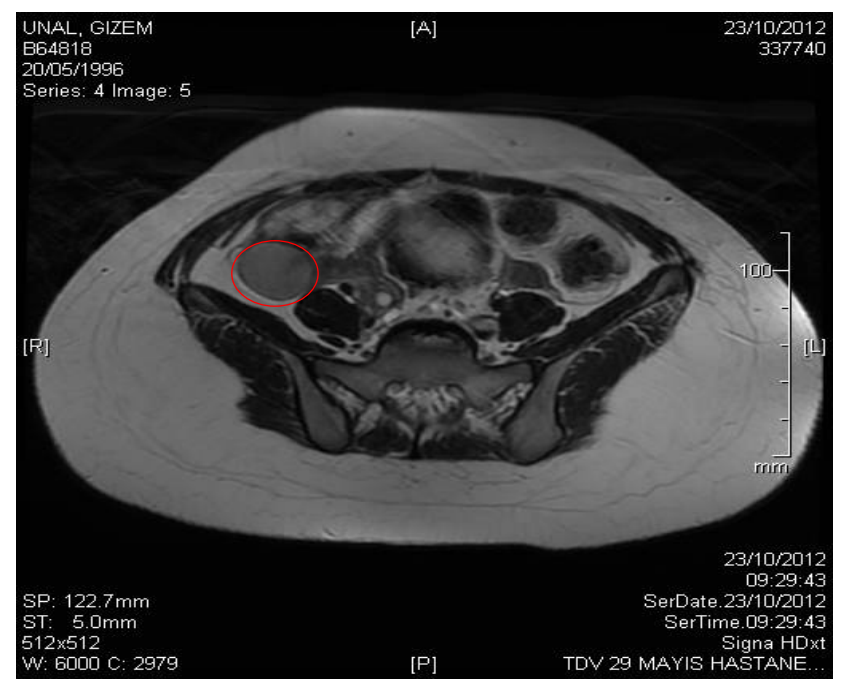

Figure 2: Peritoneal hemorrhage.
During the 5-8th weeks of gestation, sexual differentiation begins with the differentiation of the Wolffian (mesophrenic) and Mullerian (paramesophrenic) ducts. In the absence of anti-Mullerian hormone secreted by the Sertoli cells in the testes, the Mullerian duct system develops in 3 phases in the female fetus: organogenesis, fusion and resorption. Two Mullerian ducts develop in organogenesis. These ducts then fuse together, and at the $20^{\text {th }}$ week of gestation, the septum in the middle is resorbed to form the final shapes of the organs. This embryonic development results in the development of the uterus, fallopian tubes, cervix, and the upper one-third of the vagina $[3,4]$.

Mullerian duct anomalies may be accompanied by urinary system anomalies since both systems go through a common developmental process [5]. There were no urinary system anomalies in the current case.

The American Fertility Society (AFS) classified Mullerian anomalies in seven groups in 1998 [6] (Figure 3). However, reports of cases unidentified in this classification exist in the literature [6,7]. The anomaly found in the current case was not identified in this classification system. The patient had undergone surgery with a diagnosis of hemorrhagic cyst at the age of 12 years due to peritoneal bleeding. USG was insufficient in clarifying the case but, evaluation of MRI results by experienced specialists did shed light on the etiology of the disorder. Reporting of suchrare anomalies are important for correct diagnosis and treatment of patients.

The elevated CA-125 levels in the patient was believed to be due to chronic irritation caused by bleeding to peritone CA-125 levels

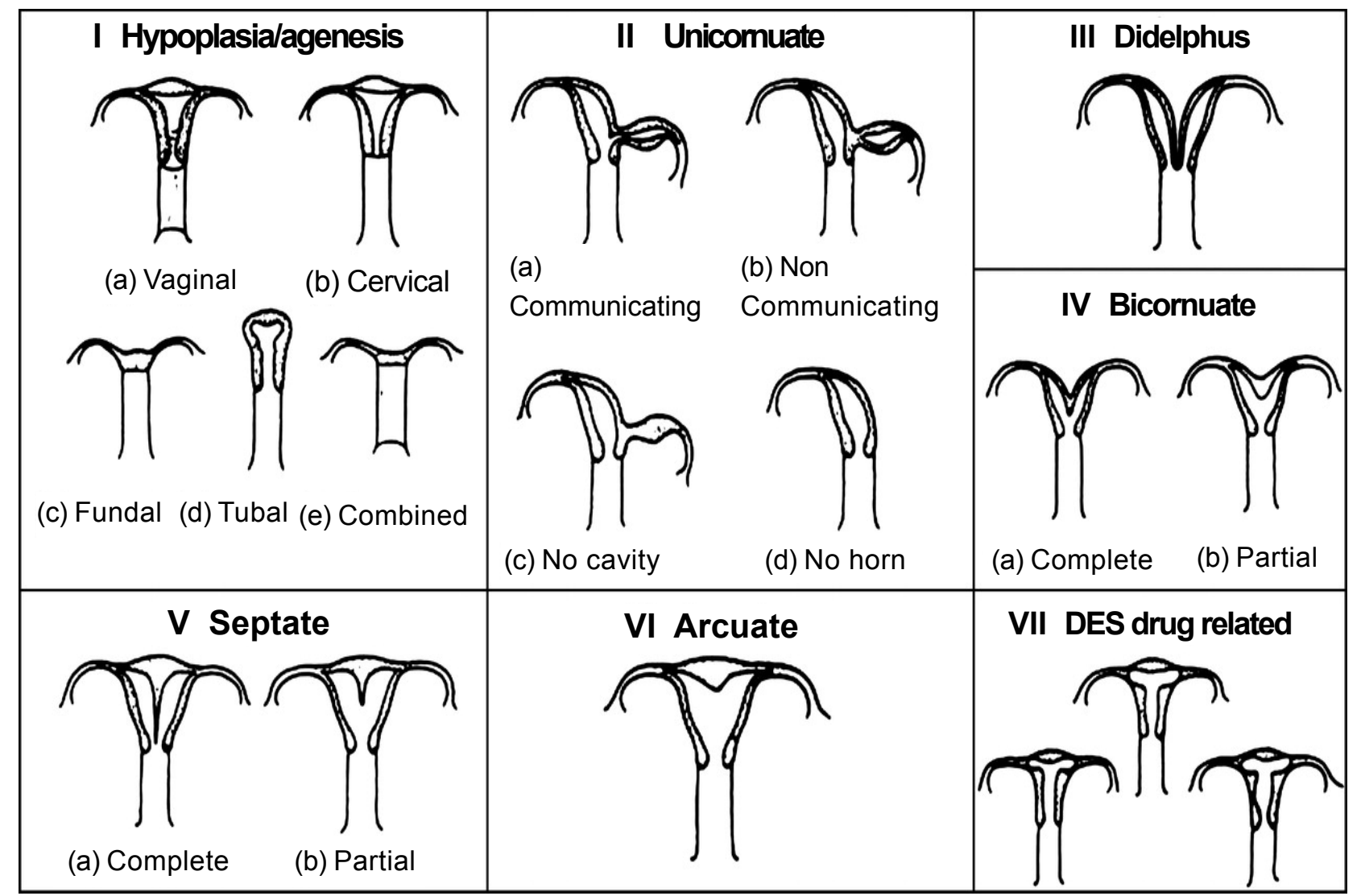

Figure 3: Classification of mullerian anomalies determined by the American Fertility Society. 
Citation: Sagsak E, Onder A, Ocal FD, Tasci Y, Agladioglu SY, et al. (2014) Primary Amenorrhea Secondary to Mullerian Anomaly. J Clin Case Rep S1: 007. doi:10.4172/2165-7920.S1-007

can rise in gynecologic and non-gynecologic cancers, particularly in ovarian cancer. Furthermore, CA-125 elevation may also be observed in benign events such as menstruation, gestation and endometriosis, pelvic inflammatory disease, and collagen tissue disease [8]. It was first shown by Bergmann et al. in 1987 that CA-125 could be a marker for peritoneal irritation and inflammation [9]. In the literature, there was only one case report with a Mullerian anomaly and CA-125 elevation. An adnexal mass, along with elevated levels of CA-125, was determined in a 15-year-old female patient that presented with a complaint of abdominal pain. Laparoscopic evaluation revealed a unicornuate uterus and hematometra in non-communicating uterine horn (AFS classification type $2 \mathrm{~b}$ ). Surgical intervention resulted in rapid normalization of CA-125 levels. Thus, in that case, CA-125 elevation was associated with Mullerian anomaly [10]. We concluded that, CA125 elevation in our patient may be explainedby recurrent menstrual bleeding to peritoneal cavity.

In conclusion, to the best of our knowledge, the described Mullerian anomaly in our case has not been reported to date. It is an additional anomaly to classification of MRKH by the AFS. All cases of primary amenorrhea with normal development of secondary sex characteristics in $46, \mathrm{XX}$ patientsshould be routinely and carefully investigated by multidisciplinary approach.

\section{References}

1. Vallerie AM, Breech LL (2010) Update in Müllerian anomalies: diagnosis, management, and outcomes. Curr Opin Obstet Gynecol 22: 381-387.
2. Demoulin A, Jouan C, Gerday C, Dubois M (1991) Pregnancy rates after transfer of embryos obtained from different stimulation protocols and frozen at either pronucleate or multicellular stages. Hum Reprod 6: 799-804.

3. Breech LL, Laufer MR (2009) Müllerian anomalies. Obstet Gynecol Clin North Am 36: 47-68

4. Acién P (1992) Embryological observations on the female genital tract. Hum Reprod 7: 437-445.

5. Grimbizis GF, Campo R (2010) Congenital malformations of the female genital tract: the need for a new classification system. Fertil Steril 94: 401-407.

6. Tanaka Y, Koyama S, Kobayashi M, Kubota S, Nakamura R, et al. (2013) Complex Müllerian malformation without any present classification: unilatera ovarian and tubal absence with an arcuate uterus. Asian J Endosc Surg 6: $55-57$.

7. Kumar S, Satija B, Wadhwa L (2012) Complex mullerian duct anomaly in a young female with primary amenorrhoea, infertility, and chronic pelvic pain. $J$ Hum Reprod Sci 5: 295-297.

8. Hovig E, Rye PD, Warren DJ, Nustad K (2001) CA 125: the end of the beginning Tumour Biol 22: 345-347.

9. Bergmann JF, Bidart JM, George M, Beaugrand M, Levy VG, et al. (1987) Elevation of CA 125 in patients with benign and malignant ascites. Cancer 59 : 213-217.

10. Basak S, MacDougal J, Prentice A (2009) A diagnostic dilemma: functioning noncommunicating uterine horn and elevated levels of CA125. J Pediatr Adolesc Gynecol 22: e127-129.
This article was originally published in a special issue, Obstetrics and Gynecology handled by Editor. Dr. Yigit Cakiroglu, Kocaeli University, Turkey 